\title{
Exceptionally slow dephasing of electronic continuum states in a semiconductor
}

\author{
P. Leisching, T. Dekorsy, H. J. Bakker, and H. Kurz \\ Institut für Halbleitertechnik, Rhenisch-Westfälische Technische Hochschule Aachen, D-52056 Aachen, Germany \\ K. Köhler \\ Fraunhofer-Institut für Angewandte Festkörperphysik, D-79108 Freiburg, Germany
}

\begin{abstract}
The coherent excitation of excitonic and continuum Wannier-Stark states in a semiconductor superlattice with femtosecond laser pulses leads to a modulation of both the interband and the intraband polarization. The field dependence of the Bloch oscillation frequency of the nonlinear interband polarization is shown to differ strongly from that of the intraband polarization. We find that the observed modulation of the intraband polarization results from the superposition of electronic continuum states. This implies that the intraband dephasing time of these continuum states is exceptionally long.
\end{abstract}

The observation of quantum-beat phenomena in semiconductors and semiconductor heterostructures is a subject of great current interest. ${ }^{1}$ The quantum-beat phenomena observed cover a wide variety of excitations like beats of light-hole and heavy-hole excitons, ${ }^{2,3}$ of bound and free excitons, ${ }^{4}$ of coupled states in double quantum wells, ${ }^{5,6}$ of excitons and biexcitons in quantum wells, ${ }^{7}$ and of Wannier-Stark (WS) states in superlattices, ${ }^{8-11}$ so-called Bloch oscillations (BO's). Several experimental techniques have been developed for the observation of the dynamics of coherently excited wave packets. Among these, the technique of transient four-wave mixing (FWM) is by far the most prominent. FWM signals arise from the third-order interband polarization and are predominantly of an excitonic nature. The charge oscillations associated with the wave packet dynamics can also be investigated with $\mathrm{THz}$ emission spectroscopy ${ }^{3,6,10}$ and electro-optic sampling. ${ }^{11}$ These techniques provide complementary information to FWM, because they probe the dynamics of the intraband polarization. All these techniques rely on the availability of femtosecond laser pulses, because the coherence in III-V compound semiconductors decays within a few picoseconds.

BO's in semiconductor superlattices have been observed in different time-resolved optical experiments employing femtosecond laser pulses. The broadband spectrum of these pulses excites a coherent superposition of several WS states, ${ }^{12,13}$ resulting in the observation of quantum beats with the BO frequency in FWM. ${ }^{8,9} \mathrm{BO}$ 's also lead to the emission of $\mathrm{THz}$ radiation, which implies that the intraband polarization is modulated by the oscillation of the excited electronic wave packets relative to the localized holes. ${ }^{10}$ These results have stimulated further experimental ${ }^{11,14,15}$ and theoretical work concerning the nature of the most relevant dephasing mechanisms ${ }^{14,16}$ and the nature of the excited states. ${ }^{17,18}$ Dignam et al. ${ }^{17}$ pointed out that optically excited BO's are of excitonic character, i.e., that their dynamics is strongly influenced by the Coulomb interaction of the optically excited electron-hole pairs. The frequency and the macroscopic dipole associated with the BO's is found to depend on the exact excitation conditions. They considered the contribution of continuum states performing BO's to be of minor importance, due to the rapid dephasing of these states. ${ }^{19,20}$ For narrow miniband superlattices, Meier et al. ${ }^{18}$ derived from the numerical solution of the semiconductor Bloch equations that significant differences can arise between the $\mathrm{BO}$ frequencies that would be observed in $\mathrm{FWM}$ and $\mathrm{THz}$ emission experiments. In another theoretical study ${ }^{21}$ it was shown that the $\mathrm{THz}$ signal emitted from biased double-quantum-well structures contains frequency components related to both excitonic and continuum states. An experimental verification of these predictions is difficult because the observed modulation frequency sensitively depends on the exact excitation conditions ${ }^{15}$ and FWM and $\mathrm{THz}$ spectroscopy have to be performed in different experimental geometries and with different excitation conditions. ${ }^{9,10}$

In this paper, we report on a comparative study of the coherent dynamics of the interband and the intraband polarization of BO's in biased $\mathrm{GaAs} / \mathrm{Al}_{x} \mathrm{Ga}_{1-x} \mathrm{As}$ superlattices by the simultaneous performance of FWM and transmittive electro-optic sampling (TEOS). ${ }^{11}$ In contrast to THz-emission spectroscopy, TEOS can be performed in exactly the same experimental arrangement as FWM. TEOS enables us to monitor directly the dynamics of the intraband dipole moment induced by the spatial motion of coherent electronic wave packets. This method is based on the detection of the optical anisotropy induced via the Pockels effect by the macroscopic longitudinal polarization parallel to the growth direction. We observe that the modulation frequencies of the interband and intraband polarization are significantly different and that this difference strongly depends on the applied electric field. We find strong evidence that the intraband polarization observed in TEOS mainly results from the coherent superposition of electronic continuum states. This implies that these states have an exceptionally long intraband dephasing time. We find that this dephasing is even slower than the excitonic interband dephasing.

The laser source used in the experiments is a Kerr-lens mode-locked Ti:sapphire laser that delivers pulses with 
$130 \mathrm{fs}$ pulse duration and a spectral width of $22 \mathrm{meV}$. TEOS is a conventional pump-probe technique in transmission geometry, where a circularly polarized probe pulse is analyzed polarization sensitively as a function of the time delay with respect to a stronger, linearly polarized pump-pulse. The detected transmission changes are proportional to changes in the longitudinal intraband polarization $P_{z} \cdot{ }^{11}$ In FWM, the time-integrated signal generated in the background-free direction $2 k_{2}-k_{1}$ is recorded with a photomultiplier, as a function of the time delay between the pulses in the directions $k_{1}$ and $k_{2}$. This signal is directly proportional to the square of the third-order interband polarization $\left|P^{(3)}\right|^{2}$. In order to perform TEOS and FWM experiments under equal conditions, FWM is also performed with one beam weaker in intensity and circularly polarized, i.e., the probe beam in TEOS. To switch between the two experiments, only the input cable to the data-acquisition system has to be changed.

The superlattice consists of 35 periods of $97 \AA$ wide GaAs wells and $17 \AA$ wide $\mathrm{Al}_{0.3} \mathrm{Ga}_{0.7}$ As barriers embedded between intrinsic $\mathrm{Al}_{0.3} \mathrm{Ga}_{0.7} \mathrm{As}$ buffers. This structure with a total intrinsic region of $1 \mu \mathrm{m}$ is grown on an $n$-doped substrate. An external bias can be applied via an Ohmic contact on the substrate and a semitransparent $\mathrm{Cr} / \mathrm{Au}$ Schottky contact on top of the sample. For transmission measurements, the substrate has been partially removed by wet etching. The sample is positioned in a cryostat and the measurements are performed at $10 \mathrm{~K}$. For the lowest electronic miniband, a minibandwidth of $\Delta=19 \mathrm{meV}$ is calculated using the Kronig-Penny model. The small heavy-hole minibandwidth of $1.9 \mathrm{meV}$ leads to a strong localization of the heavy-hole wave functions, even at small electric fields. The WS ladder of the sample has been characterized by photocurrent and transmission spectroscopy. ${ }^{15}$ The laser wavelength is centered at 798 $\mathrm{nm}$ between the heavy-hole 0 to electron $0 \mathrm{WS}$ transition $(h 0-e 0)$ and the heavy-hole 0 to electron -1 WS transition $(h 0-e-1)$.

In Fig. 1, TEOS and FWM data are compared for an applied voltage of $-0.95 \mathrm{~V}$. The excitation density is $2 \times 10^{9}$ carriers per $\mathrm{cm}^{2}$ and well. The FWM signal is plotted on a logarithmic scale and the TEOS data on a linear one. The interband dephasing time $T_{2}^{\text {inter }}$ of the nonlinear polarization leading to the FWM signal is approximately $1 \mathrm{ps}$. The relatively high initial peak compared to the oscillatory part of the signal results from the excitation of continuum states that have an interband dephasing time below $100 \mathrm{fs}^{20}$ The TEOS data exhibit a steplike drop at zero time delay, due to an instantaneously created polarization in the $z$ direction ${ }^{6,17}$ and an additional coherence peak that cannot be avoided in this geometry. ${ }^{11}$ The signal is strongly modulated due to BO's, which are clearly observable for more than 3 ps. The intraband dephasing time constant is equal to $2.4 \mathrm{ps}$, as determined by a numerical fit to the oscillations. The oscillation frequencies are determined by an accurate determination of the spacing of the maxima and minima in the FWM signal and by a fast-Fourier-transform of the TEOS data. ${ }^{11}$ Surprisingly, the frequencies are significantly different: $h \nu_{\mathrm{BO}}^{\mathrm{TEOS}}=(10.7 \pm 0.2) \mathrm{meV}$ and $h \nu_{\mathrm{BO}}^{\mathrm{FWM}}$

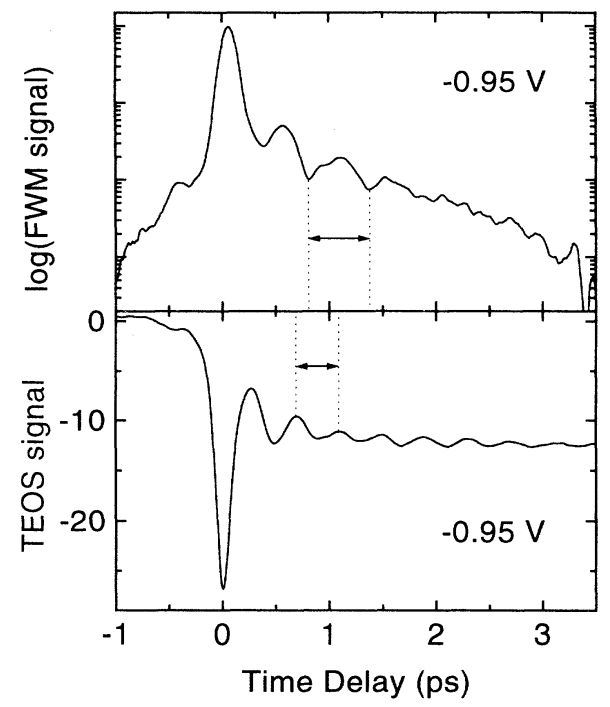

FIG. 1. Simultaneously recorded TEOS (linear plot) and FWM (semilogarithmic plot) signals at a voltage of -0.95 $\mathrm{V}$, applied to a $\mathrm{GaAs} / \mathrm{Al}_{0.3} \mathrm{Ga}_{0.7} \mathrm{As}$ superlattice at a lattice temperature of $10 \mathrm{~K}$. The excitation density is $2 \times 10^{9} \mathrm{~cm}^{-2}$ carriers per well. The arrows indicate the oscillation period in each experiment.

$=(9.1 \pm 0.6) \mathrm{meV}$. The larger error in the determination of the BO frequency in FWM is due to the smaller number of oscillation periods within the dephasing. To investigate the field dependence of the modulation frequencies, we tune the reverse bias from -0.4 to -1.0 V. Figure 2 shows the oscillation energies obtained from FWM and TEOS experiments, as a function of reverse bias. At higher electric fields, the frequencies become strongly different. The maximum energy difference measured is $2.2 \mathrm{meV}$, at a bias of $-1.0 \mathrm{~V}$.

For BO's, a linear relation between frequency and field is expected, given by the semi-classical relation $\nu_{\mathrm{BO}}=e F d / h$. We do not observe a strictly linear dependence of the frequency on the field in either FWM or TEOS. This deviation from linearity results from the dependence of the absorption on the reverse bias. The

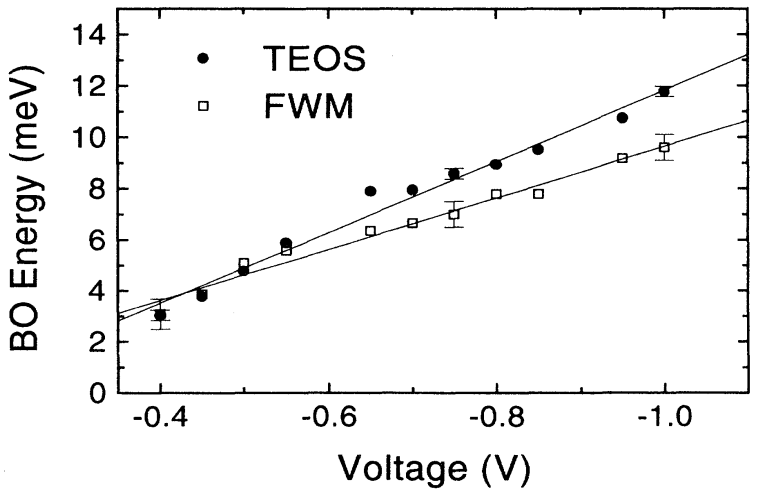

FIG. 2. Energy of the Bloch oscillations, as a function of the applied voltage obtained from the FWM data (open squares) and TEOS data (solid circles). The straight lines are linear fits to the data. 
strength of the absorption determines the density of excited carriers and thereby the amount of screening of the applied electric field. ${ }^{11,15}$ Nevertheless, the data can be fitted well by a straight line with a slope of $13.8 \mathrm{meV} / \mathrm{V}$ for TEOS and $10.4 \mathrm{meV} / \mathrm{V}$ for FWM. The theoretical slope expected from $\nu_{\mathrm{BO}}=e F d / h$ is $11.4 \mathrm{meV} / \mathrm{V}$, when it is assumed that the voltage drops completely over the full intrinsic region of the sample. In the experiment, a somewhat larger slope is expected, due to the voltage dependence of the screening effect that results from carrier accumulation. Unfortunately, this accumulation of carriers cannot be avoided at the high laser repetition rate of $80 \mathrm{MHz}$. At a large reverse bias of -1.7 $\mathrm{V}$, outside the range depicted in Fig. 2, we observe in TEOS experiments, oscillations up to $21 \mathrm{meV}$ energy. It is difficult to obtain reliable values for the frequency of the interband polarization in this region with FWM, because the dephasing of the nonlinear interband polarization becomes too fast under these excitation conditions. However, when we extrapolate the BO energy in FWM towards the voltage of $-1.7 \mathrm{~V}$, a value of $h \nu_{\mathrm{BO}}^{\mathrm{FWM}}=17.5$ $\mathrm{meV}$ is obtained, which is $3.5 \mathrm{meV}$ smaller than the oscillation energy observed in TEOS.

In the following, we will show that the observed frequency difference results from the difference in the relative contribution of excitons to the interband and intraband polarization. Previous investigations showed that the signals observed in FWM on semiconductor heterostructures dominantly result from the nonlinear interband polarization of excitons. ${ }^{15,17}$ For excitonic wave packets, the oscillation frequency will depend sensitively on the excitonic binding energies of the transitions involved, as shown in Fig. 3. At higher fields, the generated wave packet is formed by the superposition of $h 0-e 0$ and $h 0-e-1$ WS states. At fields $F \approx \Delta / e d$, the electronic WS wave functions become strongly localized leading to an increase of the absorption strength and the excitonic binding energy $E_{B}^{(h 0, e 0)}$ of the $h 0-e 0$ transition, while the $h 0-e-1$ transition becomes "indirect," leading to a reduction of the excitonic binding energy $E_{B}^{(h 0, e-1)}$. Therefore,

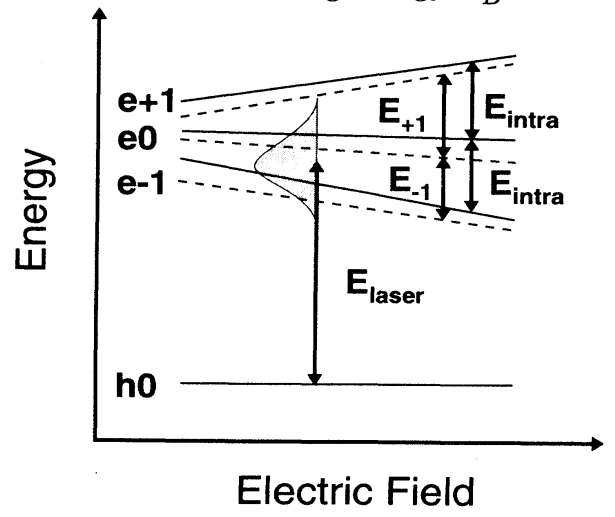

FIG. 3. Illustration (not to scale) of the field dependence of the $h 0-e-1, h 0-e 0$, and $h 0-e+1$ Wannier-Stark continuum (solid lines) and exciton transitions (dashed lines) at higher fields. The energy splitting between continuum states is denoted as $E_{\text {intra }}$, between the $h 0-e 0$ and $h 0-e-1$ excitons as $E_{-1}$, and between the $h 0-e 0$ and $h 0-e+1$ excitons as $E_{+1}$. $E_{\text {laser }}$ is the laser energy. Note that $E_{-1}<E_{\text {intra }}<E_{+1}$. the excitonic BO frequency is expected to increase less with increasing electric field than the $\mathrm{BO}$ frequency that would result from the superposition of electronic continuum states. A superposition of electronic continuum states will follow the semiclassical frequency/field relation $\nu_{\mathrm{BO}}=e F d / h$. The deviation from this relation is given by the difference in the excitonic binding energies $E_{B}^{(h 0, e 0)}-E_{B}^{(h 0, e-1)}$. If the initially created wave packet would consist of a superposition of the $h 0-e 0$ and $h 0-e+1$ WS states, the frequencies in FWM are expected to be larger compared to $\nu_{\mathrm{BO}}=e F d / h$ by $E_{B}^{(h 0, e 0)}-E_{B}^{(h 0, e+1)}$. For a quantitative determination of the excitonic effects, the excitonic binding energies are calculated numerically for different electric-field strengths. The excitonic wave functions and energies are obtained from a variational calculation for the in-plane excitonic Bohr radius. ${ }^{22}$ The out-of-plane part of the wave functions of the electrons $(e 0, e-1)$ and the holes $(h 0)$ is calculated numerically via a Numerov algorithm. ${ }^{15}$ In this calculation of the binding energy, the coupling of the miniband states is neglected so that this calculation will not yield accurate results at very low fields $(<4 \mathrm{kV} / \mathrm{cm})$. However, at higher values of the electric field in our experiment, the miniband states are decoupled and transform into WS states, so that the calculated values become quite accurate. In Fig. 4(a) the calculated exciton binding energies $E_{B}^{(h 0, e 0)}$ and $E_{B}^{(h 0, e-1)}$ are plotted as a function of the applied electric field. The
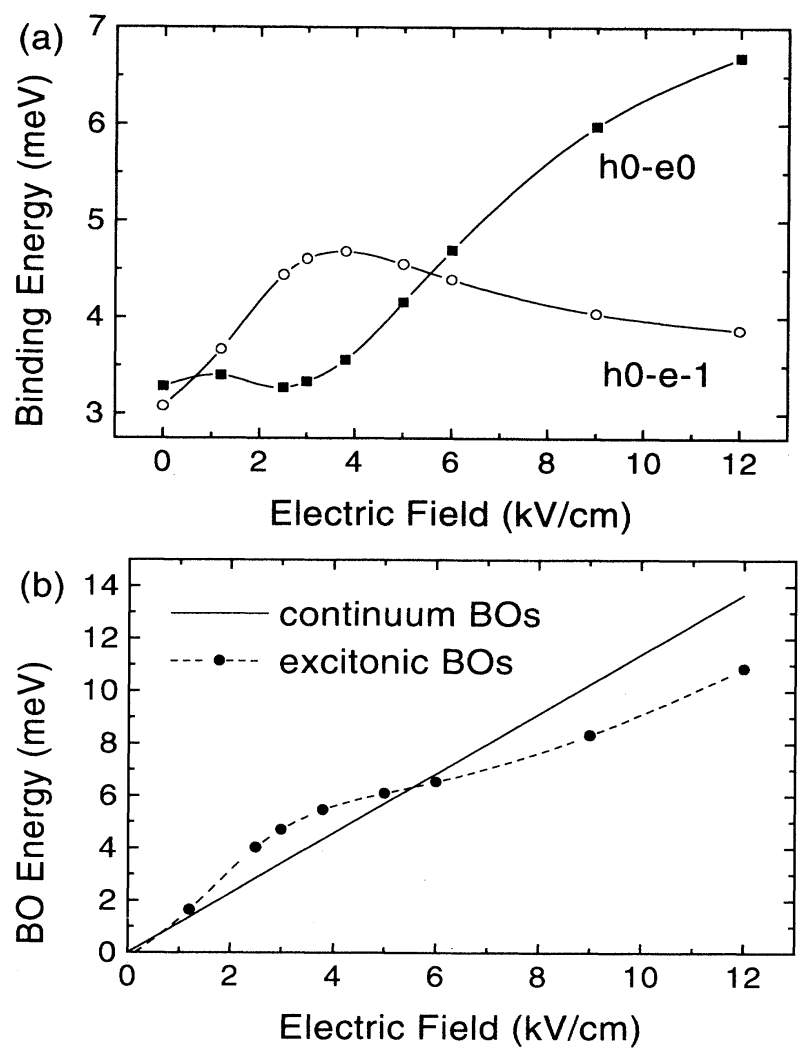

FIG. 4. (a) Calculated field dependence of the excitonic binding energies of the $h 0-e 0$ (solid squares) and $h 0-e-1$ (open circles) Wannier-Stark transitions. (b) Energies of Bloch oscillations calculated using Fig. 4 (a) (solid circles) and the semiclassical frequency dependence $h \nu_{\mathrm{BO}}=e F d$. 
value of $E_{B}^{(h 0, e 0)}$ increases from approximately $3 \mathrm{meV}$ to $8 \mathrm{meV}$, whereas $E_{B}^{(h 0, e-1)}$ decreases from $4.5 \mathrm{meV}$ to 3.5 $\mathrm{meV}$. In Fig. 4(b), the calculated energy of the excitonic BO's is presented and compared to $h \nu_{\mathrm{BO}}=e F d$.

We observe that at a small reverse bias of $-0.5 \mathrm{~V}$, corresponding to an electric field $F_{\text {exp }}=4.4 \mathrm{kV} / \mathrm{cm}$, the modulation frequency of the interband polarization observed with FWM is slightly higher than the modulation frequency of the intraband polarization observed with TEOS. The field in the sample $F_{\exp }$ is calculated from $h \nu_{\mathrm{BO}}^{\mathrm{TEOS}} / e d$. The relatively high frequency of the interband polarization at small bias can be explained from the fact that at this bias, the excitonic binding energy $E_{B}^{(h 0, e-1)}$ is larger than $E_{B}^{(h 0, e 0)}$. At a higher bias of $-0.95 \mathrm{~V}$, corresponding to $F_{\exp }=9.4 \mathrm{kV} / \mathrm{cm}$, the frequency of the interband polarization is $1.6 \mathrm{meV}$ lower than that of the intraband polarization. For this bias, the modulation frequency of the interband polarization is relatively small because $E_{B}^{(h 0, e-1)}$ is much smaller than $E_{B}^{(h 0, e 0)}$. These results show that the modulation frequency of the intraband polarization is hardly influenced by excitonic effects and closely follows the semiclassical frequency/field relation if the field-dependent carrieraccumulation effects are taken into account. Hence, we conclude that the observed modulation of the intraband polarization results from the superposition of electronic continuum states. This has two important consequences. First, the intraband dephasing time of electronic continuum states must be exceptionally long ( $2.4 \mathrm{ps})$ and is much longer than their interband dephasing time $(<100$ fs). Second, the femtosecond laser pulse apparently excites predominantly continuum WS states. Therefore, the observation of strong excitonic character in the mod- ulation of the interband polarization is solely due to the fact that the initially dominant interband polarization of the continuum states decays within $100 \mathrm{fs}$. The contribution of the continuum states to the interband polarization causes the strong instantaneous signal observed in FWM.

In a recent theoretical study on BO's in a semiconductor superlattice, it was predicted that electron-hole interactions play an important role for the interband polarization and hardly influence the intraband polarization. ${ }^{18}$ This agrees very well with our experimental findings: the observed modulation of the intraband polarization results from the coherent superposition of electronic continuum states and is thus hardly effected by electron-hole interactions. An important implication of this result is that the observation of a long-living electronic coherence in semiconductors is not necessarily related to the stability of excitons at low lattice temperatures.

In conclusion, we reported on the observation of a distinct difference in the modulation frequency of the interband and intraband polarization of Bloch oscillations in a GaAs $/ \mathrm{Al}_{0.3} \mathrm{Ga}_{0.7} \mathrm{As}$ superlattice. This difference forms conclusive proof for the absence of excitonic effects in the intraband polarization. Hence, the Bloch oscillations of the intraband polarization, as observed with TEOS, result from the coherent superposition of electronic continuum states. This means that the intraband dephasing of electronic continuum states is extremely slow and is even slower than the interband dephasing of excitons.

We thank H. G. Roskos for useful and stimulating discussions. W. Beck and R. Ott are acknowledged for experimental assistance. This work was supported by the Volkswagen Stiftung and the Deutsche Forschungsgemeinschaft.
${ }^{1}$ See, e.g., contributions to Coherent Optical Interactions in Semiconductors, Vol. 330 of NATO Advanced Study Institute, Series B: Physics, edited by R.T. Philips (Plenum Press, New York, 1994).

${ }^{2}$ K. Leo, T.C. Damen, J. Shah, E.O. Göbel, and K. Köhler, Appl. Phys. Lett. 57, 19 (1990).

${ }^{3}$ P.C.M. Planken, M.C. Nuss, I. Brenner, K.W. Goosen, M.S.C. Luo, S.L. Chuang, and L. Pfeifer, Phys. Rev. Lett. 69, 3800 (1992).

${ }^{4}$ K. Leo, T.C. Damen, J. Shah, and K. Köhler, Phys. Rev. B 42, 11359 (1990).

${ }^{5}$ K. Leo, J. Shah, E.O. Göbel, T.C. Damen, S. SchmittRink, W. Schäfer, and K. Köhler, Phys. Rev. Lett. 66, 201 (1991).

${ }^{6}$ H.G. Roskos, M.C. Nuss, J. Shah, K. Leo, D.A.B. Miller, A.M. Fox, S. Schmitt-Rink, and K. Köhler, Phys. Rev. Lett. 68, 2216 (1992).

${ }^{7}$ D.J. Lovering, R.T. Philips, G.J. Denton, and G.W. Smith, Phys. Rev. Lett. 68, 1880 (1992).

${ }^{8}$ J. Feldmann, K. Leo, J. Shah, D.A.B. Miller, J. E. Cunningham, S. Schmitt-Rink, T. Meier, G. von Plessen, A. Schulze, and P. Thomas, Phys. Rev. B 46, 7252 (1992).

${ }^{9}$ K. Leo, P. Haring Bolivar, F. Brüggemann, R. Schwedler, and K. Köhler, Solid State Commun. 84, 943 (1992).

${ }^{10}$ C. Waschke, H.G. Roskos, R. Schwedler, K. Leo, H. Kurz, and K. Köhler, Phys. Rev. Lett. 70, 3319 (1993).

${ }^{11}$ T. Dekorsy, P. Leisching, K. Köhler, and H. Kurz, Phys.
Rev. B 50, 8106 (1994).

${ }^{12}$ E.E. Mendez, F. Agulló-Rueda, and J.M. Hong, Phys. Rev. Lett. 60, 2426 (1988).

${ }^{13}$ P. Voisin, J. Bleuse, C. Bouche, S. Gaillard, C. Alibert, and A. Regreny, Phys. Rev. Lett. 61, 1639 (1988).

${ }^{14}$ G. von Plessen, T. Meier, J. Feldmann, E.O. Göbel, P. Thomas, K.W. Goosen, J.M. Kuo, and R.F. Kopf, Phys. Rev. B 49, 14058 (1994).

${ }^{15}$ P. Leisching, P. Haring Bolivar, W. Beck, Y. Dhaibi, K. Leo, K. Köhler, and H. Kurz, Phys. Rev. B 50, 14389 (1994).

${ }^{16}$ A. M. Bouchard and M. Luban, Phys. Rev. B 47, 6815 (1993).

${ }^{17}$ M. Dignam, J.E. Sipe, and J. Shah, Phys. Rev. B 49, 10502 (1994).

${ }^{18}$ T. Meier, G. von Plessen, P. Thomas, and S.W. Koch, Phys. Rev. Lett. 73, 902 (1994).

19 D.-S. Kim, J. Shah, J.E. Cunningham, T.C. Damen, W. Schäfer, M. Hartmann, and S. Schmitt-Rink, Phys. Rev. Lett. 68, 1006 (1992).

${ }^{20}$ A. Lohner, K. Rick, P. Leisching, A. Leitensdorfer, T. Elsaesser, T. Kuhn, F. Rossi, and W. Stolz, Phys. Rev. Lett. 71, 77 (1993).

${ }^{21}$ T. Kuhn, E. Binder, and G. Mahler, Phys. Rev. B 50, 18319 (1994).

${ }^{22}$ R.H. Yan, F. Laruelle, and L.A. Coldren, Appl. Phys. Lett. 55, 2002 (1989). 


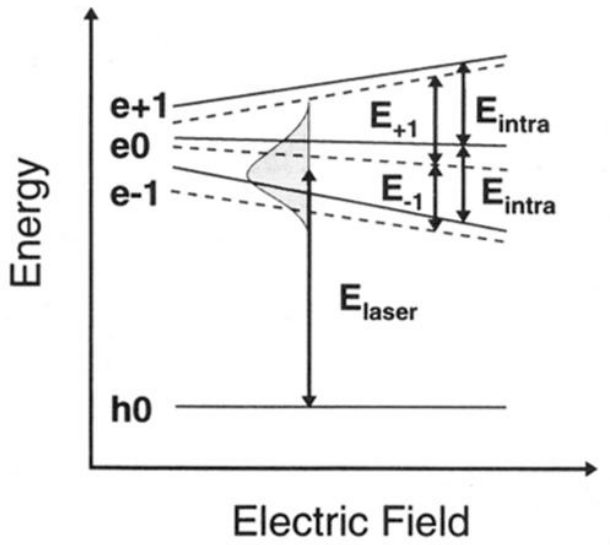

FIG. 3. Illustration (not to scale) of the field dependence of the $h 0-e-1, h 0-e 0$, and $h 0-e+1$ Wannier-Stark continuum (solid lines) and exciton transitions (dashed lines) at higher fields. The energy splitting between continuum states is denoted as $E_{\text {intra }}$, between the $h 0-e 0$ and $h 0-e-1$ excitons as $E_{-1}$, and between the $h 0-e 0$ and $h 0-e+1$ excitons as $E_{+1}$. $E_{\text {laser }}$ is the laser energy. Note that $E_{-1}<E_{\text {intra }}<E_{+1}$. 